\title{
Pomegranate seed oil consumption during a period of high-fat feeding reduces weight gain and reduces type 2 diabetes risk in CD-1 mice
}

\author{
Brian K. McFarlin ${ }^{1,2 *}$, Kelley A. Strohacker ${ }^{1}$ and Michael L. Kueht ${ }^{1}$ \\ ${ }^{1}$ Laboratory of Integrated Physiology, Department of Health and Human Performance, University of Houston, 3855 Holman Street, \\ 104U Garrison, Houston, TX 77204-6015, USA \\ ${ }^{2}$ Texas Obesity Research Center, University of Houston, 110 Garrison, Houston, TX 77204, USA
}

(Received 9 July 2008 - Revised 9 October 2008 - Accepted 10 October 2008 - First published online 16 December 2008)

The health benefits of pomegranate consumption have recently received considerable scientific focus, with most studies examining fruit and/or juice consumption. Pomegranate seed oil (POMo) is a rich source of 9-cis, 11-trans conjugate linolenic acid (CLA), which may offset the side-effects associated with weight gain. Male, wild-type CD-1 mice were divided into one of three groups (twenty per group): high-fat (HF), $\mathrm{HF}+$ seed oil (HF + POMo) or lean control (LN). In HF and HF + POMo, mice were provided access ad libitum to a high-fat chow (60\% of energy from fat). HF + POMo was supplemented with $61.79 \mathrm{mg} \mathrm{POMo} / \mathrm{d}$. LN consumed a restricted low-fat (10\% of energy from fat) chow to maintain body weight within $5 \%$ of initial weight. Plasma was analysed for biomarkers associated with cholesterol profile (total cholesterol, HDL and TAG), glucose sensitivity (glucose and insulin), adipose tissue accumulation (leptin and adiponectin) and systemic low-grade inflammation (C-reactive protein and haptoglobin). The key findings of this study were that weight gain was associated with an increase in biomarkers of cholesterol profile, glucose sensitivity, adipose tissue accumulation and systemic low-grade inflammation $(P<0 \cdot 05)$. POMo only altered body weight accumulation, final body weight, leptin, adiponectin and insulin $(P<0 \cdot 05)$. We found that despite a similar level of energy intake, HF mice had a greater concentration of leptin and a lower concentration of adiponectin compared to HF + POMo mice. POMo intake was associated with an improvement in insulin sensitivity, suggesting that risk of developing type 2 diabetes may have been reduced; however, CVD risk did not change.

High-fat feeding: Leptin: Adiponectin: Weight gain

Body weight gain via high-fat feeding is associated with an increase in risk of developing CVD, type 2 diabetes mellitus, hypercholesterolaemia and fatty liver syndrome ${ }^{(1-4)}$. Poor diet habits and physical inactivity are the most common causes of excessive weight gain in today's society ${ }^{(4-8)}$. Consumption of pomegranate (Punica granatum, Punicaceae) fruit and/or juice has been reported to reduce the risk of CVD and prostate cancer $^{(9,10)}$. The health benefits of juice consumption have been attributed to the polyphenol concentration ${ }^{(9)}$. The present investigation expands on the known health benefits of pomegranates by examining another portion of the fruit, the seed arils. Each pomegranate contains 600-800 seeds and when purified, oil from these seeds (POMo) is a rich naturally occurring source of the bioactive compound 9-cis, 11-trans conjugate linolenic acid $(\mathrm{CLA})^{(11)}$. Since the pomegranate juice and seed oil contain different bioactive compounds, it is reasonable to speculate that they may exert different physiologic effects.

The key objective of the present investigation was to describe the potential health benefits of POMo consumption during a period of ad libitum high-fat feeding (60\% of energy from fat) designed to increase body weight. Consistent with what others have reported regarding the effects of CLA supplementation $^{(12,13)}$, we speculated that POMo supplementation may offset some, but not all of the side-effects associated with weight gain due to the interaction of the various bioactive compounds in POMo. The purpose of the present study was to determine if POMo supplementation could offset increases in cholesterol profile, insulin resistance, markers of adipose accumulation, markers of systemic inflammation and body weight/composition changes.

\begin{abstract}
Methods
Animals

All protocols used in the present study were approved by the University of Houston committee for animal care and use. We certify that all applicable institutional and governmental regulations concerning the use of animals were followed during this research. The present investigation utilized wild-type, outbred, specific pathogen-free CD-1 male mice (Charles Rivers Labs, Wilmington, MA, USA). We chose to use an outbred strain to minimize the deleterious effects of ageing, which are common to inbred mice ${ }^{(14)}$. Mice were allowed
\end{abstract}


$14 \mathrm{~d}$ to acclimate to the University of Houston animal care facility and were maintained on a standard 12:12 h lightdark cycle (lights on at 06.00 hours). Throughout the course of the study, mice were housed individually.

\section{Group assignment}

Following acclimation, mice were randomly assigned to one of the following three groups (twenty per group): high-fat feeding ( $60 \%$ of energy from fat; HF), high-fat feeding + POMo supplement (HF + POMo) or lean control (LN). The HF diet $(60 \%$ fat, $20 \%$ carbohydrate, $20 \%$ protein) was purchased from Research Diets Inc. (New Brunswick, NJ, USA). POMo for the present study was donated by POM Wonderful LLC (Los Angles, CA, USA). Purification and analytical analysis of POMo was completed by an outside laboratory (POS Pilot Plant Corp., Saskatchewan, Canada), who found the major forms of fatty acids to be as follows: $64.79 \% 18: 3$ (9,11,13-linolenic acid), $14.23 \%$ $18: 3$ (conjugated linolenic acid isomers), 6.17\% $18: 2 n-6$ (linoleic), $5.07 \%$ 18:1n-9 (oleic), $2 \cdot 16 \% \mathrm{C} 16$ (palmitic), $2.30 \% 18: 2$ (CLA isomers), $2.08 \% \mathrm{C} 18$ (steric) and trace amounts $(<1 \%)$ of other fatty acids. In order to facilitate daily POMo supplementation, the POMo was incorporated into a custom food, which was prepared by Research Diets Inc. with direction from our laboratory. POMo was dosed at $20 \mathrm{~g}$ oil $/ \mathrm{kg} \mathrm{HF}$ chow, resulting in an average consumption of $61.79 \mathrm{mg}$ POMo/d (baseline, $1.72 \mathrm{mg} / \mathrm{g}$ body mass per d; 14 weeks, $1.25 \mathrm{mg} / \mathrm{g}$ body mass perd). The POMo dose was chosen to supply a CLA amount which is consistent with what has been reported in the literature during similar interventions ${ }^{(12,13)}$. During the formulation process, lard was removed from the stock food to account for the fat content of POMo and maintain the diet at $60 \%$ of energy from fat. LN animals were allowed restricted access to a low-fat chow (10\% of energy from fat) to maintain their body weight within $5 \%$ of initial body weight following the 2 -week acclimation period. Animals in all three groups were maintained on their modified diets for a period of 14 weeks.

\section{Body weight/food intake tracking}

Body weight, food intake and water intake were measured on a bi-weekly basis using a digital scale. Bi-weekly values were averaged and reported as weekly values.

\section{Body composition assessment}

At the conclusion of the 14 weeks and $24 \mathrm{~h}$ prior to being killed, mice were scanned using MRI (EcoMRI; Houston, TX, USA) to determine lean mass, fat mass and total body water. All mice were scanned in duplicate and values were averaged for statistical and reporting purposes.

\section{Tissue collection}

At the conclusion of the study, fasted mice were injected intraperitoneally with a near-lethal dose of xylazine-ketamine solution, which incapacitated them within $3 \mathrm{~min}$ of injection. After the loss of the plantar reflex and prior to cessation of respiration, the thoracic cavity was opened and the inferior vena cava was severed. Blood was collected from the thoracic cavity and treated with EDTA to prevent clotting. Blood samples were placed on ice until plasma was separated by centrifugation and stored at $-80^{\circ} \mathrm{C}$ until additional analysis of blood metabolites. All samples were processed within $2 \mathrm{~h}$ of collection.

\section{Biomarker analysis}

Plasma was analysed for the presence of biomarkers that fit into four categories: (1) cholesterol profile (total cholesterol, HDL and TAG), (2) glucose sensitivity (glucose and insulin), (3) adipose tissue accumulation (leptin and adiponectin), and (4) systemic inflammation (haptoglobin and C-reactive protein). Cholesterol profile was determined using a modification of a standard enzymatic assay (Pointe Scientific, Canton, MI, USA). Blood glucose was determined using an automated analyser (YSI 2300 Stat Plus; YSI, Yellow Springs, MO, USA). Insulin, leptin, adiponectin, haptoglobin and C-reactive protein were measured using separate ELISA (Alpco Diagnostics; Salem, NH, USA). All measurements were completed in duplicate. One limitation of using a murine model is that you are unable to collect large enough volumes of blood to allow for the measurement of an unlimited number of analytes. As such, we selected the listed markers because they are traditionally used to assess disease risk.

\section{Statistical methods}

After samples were collected and data were analysed, quantile-quantile plots and histograms were generated to check that the data were normally distributed. Data that were not normally distributed were log-transformed prior to statistical testing (noted in the Results). Statistical testing of body mass was completed using a 3 (group: $\mathrm{HF}, \mathrm{HF}+\mathrm{POMo}$, $\mathrm{LN}) \times 14$ (time: weeks) ANOVA with repeated measures on the second factor. Significant $P$ values $(P<0.05)$ were corrected for the presence of repeated measures using the Huynh-Feldt method. For all other measures, data were analysed using a one-factor (group: $\mathrm{HF}, \mathrm{HF}+\mathrm{POMo}, \mathrm{LN}$ ) ANOVA. Location of significant effects $(P<0.05)$ was determined using separate Student $t$ tests with a Bonferroni adjustment for multiple comparisons. Data are reported as means and their standard errors.

\section{Results}

\section{Food intake, body weight and body composition}

Lean mice had significantly lower total energy intake $(P=0.003)$, daily energy intake $(P<0.001)$, total fat intake $(P<0.001)$ and daily fat intake $(P<0.001)$ than both HF and $\mathrm{HF}+\mathrm{POMo}$. No significant differences were found for body weight between the three groups at the start of the study. A significant interaction effect $(P=0.02)$ was found at week 12 , where weight gain in $\mathrm{HF}$ exceeded weight gain in both $\mathrm{HF}+\mathrm{POMo}$ and LN (Fig. 1). At the 14th week, the $\mathrm{HF}+\mathrm{POMo}$ mice had a significantly lower body weight $(P=0.002)$, absolute weight gain $(P=0.002)$ and percentage 


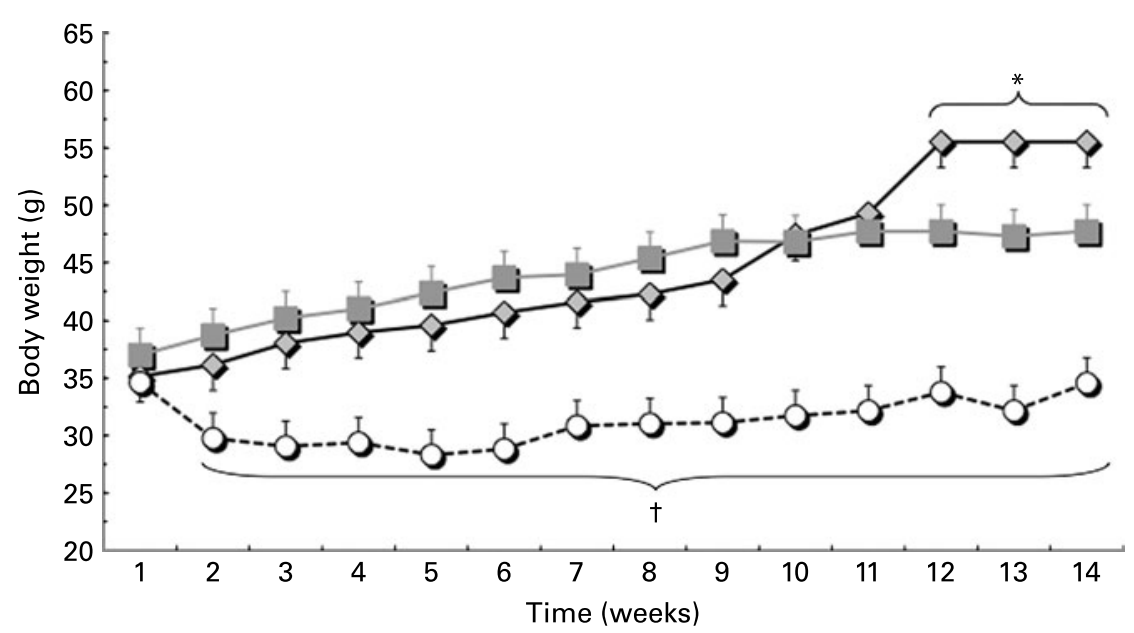

Fig. 1. Change in body weight over time for male CD-1 mice (twenty per group) provided ad libitum access to a high-fat (HF) diet (60\% of energy from fat; $\diamond)$, ad libitum access to a HF diet supplemented with pomegranate seed oil $(61.79 \mathrm{mg} / \mathrm{d}$; HF + POMo; $\square)$ or restricted access to a low-fat diet (lean control; O). Values are means with their standard errors depicted by vertical bars. Mean values were significantly greater those of the HF + POMo and control groups:

${ }^{\star} P<0.05$. Mean values were significantly less those of the $\mathrm{HF}$ and $\mathrm{HF}+\mathrm{POMo}$ groups: $\dagger P<0.05$.

weight gain $(P=0.01)$ compared to the HF mice (Fig. 1). No significant differences were found between $\mathrm{HF}$ and $\mathrm{HF}+\mathrm{POMo}$ for lean mass $(P=0 \cdot 12)$, absolute fat mass $(P=0.35)$ or percentage fat mass $(P=0.45)$.

\section{Cholesterol profile}

The measures of cholesterol profile (total cholesterol, HDL and TAG) were not normally distributed and were log-transformed prior to formal statistical testing. There was no significant difference between $\mathrm{HF}$ and $\mathrm{HF}+$ POMo for any measures of blood cholesterol profile; however, both HF and $\mathrm{HF}+\mathrm{POMo}$ had significantly greater blood concentration of total cholesterol $(P=0.004)$ and TAG $(P=0.004)$ than $\mathrm{LN}$ (Table 1 ). There were no significant differences for HDL $(P=0.71)$. All measurements were made after the 14 th week of feeding.

\section{Adipose accumulation}

ANOVA testing revealed a significant group effect for leptin $(P=0.002)$ and adiponectin $(P=0.039)$. For leptin, HF mice had $324 \%$ higher leptin than either HF + POMo or LN (Fig. 2(A)). For adiponectin, HF mice had 164\% lower levels of adiponectin than either $\mathrm{HF}+\mathrm{POMo}$ or $\mathrm{LN}$ (Fig. 2(B)).

Other investigators have reported that it is useful to examine leptin and adiponectin plasma concentration as a function of body fat mass ${ }^{(15)}$. When we completed this comparison, we found significant effects for both leptin $(P=0.019)$ and adiponectin $(P<0.001)$. With respect to leptin, $\mathrm{LN}$ was not significantly different from $\mathrm{HF}+\mathrm{POMo}$, but $\mathrm{HF}+\mathrm{POMo}$ was significantly lower than HF. A similar effect was observed for adiponectin. All measurements were made after the 14th week of feeding.

Table 1. Body composition and blood biomarker measurements in mice following 14 weeks of controlled feeding $\dagger$ (Mean values with their standard errors)

\begin{tabular}{|c|c|c|c|c|c|c|}
\hline & \multicolumn{2}{|c|}{$\mathrm{HF}$} & \multicolumn{2}{|c|}{$\mathrm{HF}+\mathrm{POMo}$} & \multicolumn{2}{|c|}{$\mathrm{LN}$} \\
\hline & Mean & SE & Mean & SE & Mean & SE \\
\hline \multicolumn{7}{|l|}{ Body composition } \\
\hline Lean mass (g) & 29.769 & 0.636 & $27 \cdot 705$ & 0.683 & $22 \cdot 782^{\star}$ & 0.658 \\
\hline Fat mass $(\mathrm{g})$ & $18 \cdot 116$ & $1 \cdot 122$ & 14.603 & $1 \cdot 164$ & $6 \cdot 023^{*}$ & 1.084 \\
\hline Body fat (\%) & $30 \cdot 71$ & 1.50 & 29.71 & 1.90 & $15 \cdot 90^{*}$ & $2 \cdot 10$ \\
\hline \multicolumn{7}{|l|}{ Cholesterol profile } \\
\hline Total cholesterol (mg/l) & 671.7 & 79.5 & 754.9 & $90 \cdot 7$ & $363.8^{*}$ & $76 \cdot 7$ \\
\hline TAG (mg/l) & $362 \cdot 6$ & $38 \cdot 1$ & 373.4 & 43.4 & $199 \cdot 1^{*}$ & $36 \cdot 7$ \\
\hline $\mathrm{HDL}(\mathrm{mg} / \mathrm{l})$ & 1175.9 & $153 \cdot 0$ & $1256 \cdot 9$ & $153 \cdot 6$ & 1073.5 & 153.5 \\
\hline \multicolumn{7}{|c|}{ Markers of systemic inflammation } \\
\hline C-reactive protein $(\mathrm{ng} / \mathrm{ml})$ & $28 \cdot 19$ & 2.03 & $27 \cdot 21$ & $2 \cdot 11$ & $24 \cdot 12$ & $2 \cdot 11$ \\
\hline Haptoglobin (ng/ml) & 62828 & 15092 & 52304 & 15828 & 25235 & 15098 \\
\hline
\end{tabular}

$\mathrm{HF}$, high-fat; LN, lean control; POMo, pomegranate seed oil.

Mean values were significantly different from those of the HF and HF + POMo groups: ${ }^{\star} P<0.05$.

†For details of procedures, see Methods. HF mice were allowed ad libitum access to a high-fat chow (60\% of energy from fat). HF + POMo mice were allowed ad libitum access to a high-fat chow supplemented with $20 \mathrm{~g} / \mathrm{kg}$ food weight of POMo. LN mice were fed a restricted, low-fat chow (10\% of energy from fat) to maintain body weight within $5 \%$ of baseline weight. 

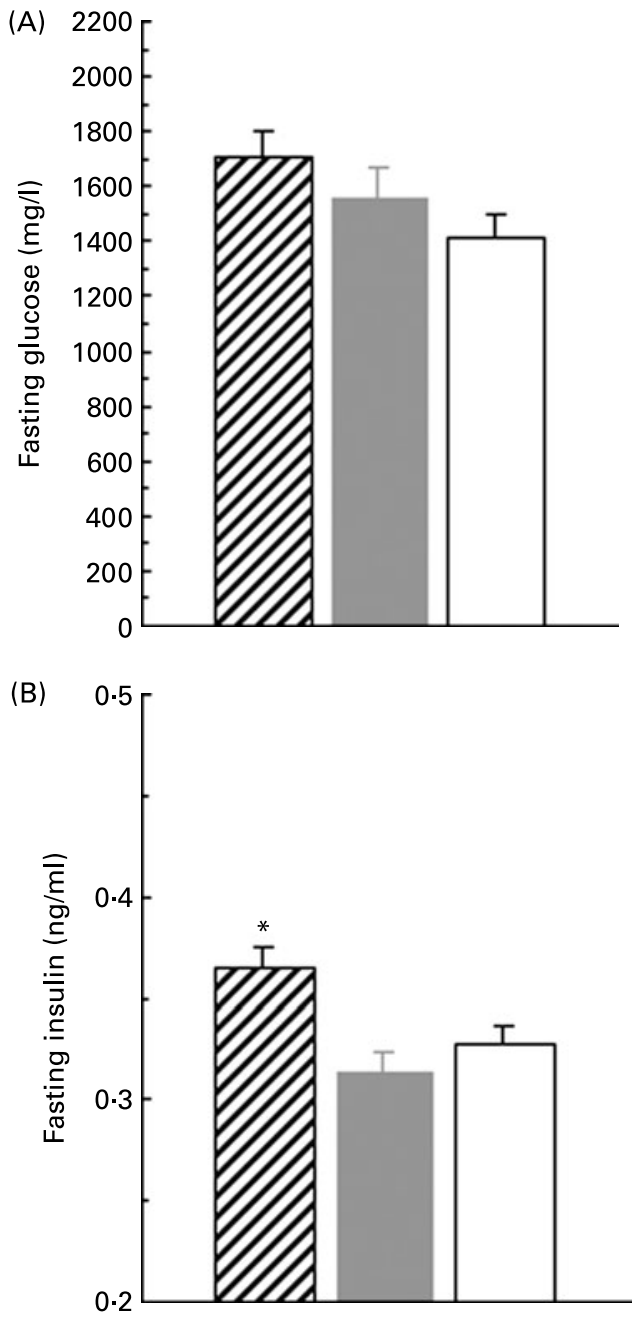

Fig. 2. Plasma leptin (A) and adiponectin (B) concentration for male CD-1 mice (twenty per group) provided ad libitum access to a high-fat (HF) diet ( $60 \%$ of energy from fat; $\mathbb{Z}$ ), ad libitum access to a HF diet supplemented with pomegranate seed oil $(61.79 \mathrm{mg} / \mathrm{d}$; HF + POMo; $\square)$ or restricted access to a low-fat diet (lean control; $\square$ ). All measurements were completed after 14 weeks of supplementation. Values are means with their standard errors depicted by vertical bars. Mean values were significantly different from those of the $\mathrm{HF}+$ POMo and control groups: ${ }^{*} P<0.05$.

\section{Glucose sensitivity}

No significant difference was found for plasma glucose concentration $(P=0 \cdot 111$; Fig. $3(\mathrm{~A}))$. A significant group effect was found for insulin concentration $(P=0.003)$, where $\mathrm{HF}$ only was significantly greater than both $\mathrm{HF}+\mathrm{POMo}$ and LN (Fig. $3(\mathrm{~B})$ ). All measurements were made after the 14th week of feeding.

\section{Systemic inflammation}

Systemic, low-level inflammation was measured via C-reactive protein and haptoglobin, which are considered to be stable blood inflammatory markers. Despite a difference in body weight between groups, we did not find a statistical difference between any of the groups for either C-reactive protein $(P=0.34)$ or haptoglobin $(P=0.45)$. All measurements were made after the 14 th week of feeding.

\section{Discussion}

The key findings of the present study were that consumption of POMo (approximately $61 \mathrm{mg} / \mathrm{d}$ ) during 14 weeks of $\mathrm{HF}$ feeding significantly reduced final body weight, percentage weight gain, leptin and insulin, and increased adiponectin compared to mice that consumed an unsupplemented $\mathrm{HF}$ diet. POMo supplementation did not significantly alter energy intake, cholesterol profile or biomarkers of systemic inflammation. Others have reported that supplementation of a diet with CLA is associated with a reduction in pro-inflammatory capacity ${ }^{(16,17)}$; however, it does not appear that POMo as a CLA source has the anti-inflammatory affect that has been reported with pure CLA supplementation. It is possible that this may be due to the interaction between the various bioactive compounds (i.e. polyphenolic acids that were not fully removed during the extraction process or an interaction among the different forms of lipid that are in purified POMo product) that are found in POMo.

Previous studies have thoroughly examined the effect of pomegranate juice consumption and have shown that the polyphenolic compounds found in the fruit significantly decrease the risk of developing CVD and prostate cancer, but not type 2 diabetes risk $^{(9,10)}$. Subsequent research has demonstrated that polyphenols have powerful antioxidant properties and this is the most likely mechanism responsible for pomegranate's protective benefits ${ }^{(18)}$. In the present study we examined POMo, which does not have polyphenols, but rather 9-cis, 11-trans CLA. We found that seed oil consumption decreased weight gain (maybe mediated by a leptin/adiponectin pathway) and insulin. The latter finding suggests that POMo consumption may have reduced risk of type 2 diabetes, while CVD risk was not changed. Given that pomegranate juice (polyphenols) and seed oil (CLA) have different bioactive compounds, it is not surprising that they exert different physiological effects.

An interesting finding of the present study was that despite similar ad libitum energy intake between the two weight-gain groups, mice supplemented with POMo gained less weight, had less leptin and had greater adiponectin concentration than mice that did not consume POMo. Leptin and adiponectin are closely related to body weight and body composition ${ }^{(15,19)}$ and the present findings provide additional support for this relationship. Fukumitsu et al. ${ }^{(19)}$ reported that addition of flaxseed lignan to a HF (30\% of energy from fat) diet in mice lowered leptin concentration and increased adiponectin. The prominent fatty acid found in flaxseed lignan is 9-cis, 11-trans CLA, which is similar to the POMo used in the present study ${ }^{(11)}$. Fu et al. ${ }^{(15)}$ reported that low plasma levels of adiponectin were associated with the development of obesity, insulin resistance and CVD. They further speculated that the changes they observed were mediated by a PPAR- $\gamma$-mediated mechanism ${ }^{(15)}$. Other investigators have reported that certain dietary seed oils have the ability to activate PPAR- $\gamma$, resulting in an alteration of adiposity, decrease in leptin and an increase in adiponectin following a period of HF feeding in mice ${ }^{(20)}$. The present findings regarding leptin and adiponectin are consistent with a PPAR- $\gamma$-mediated mechanism that others have presented. It is reasonable to speculate that POMo may counter the effects of HF feeding via a PPAR- $\gamma$-mediated mechanism. 

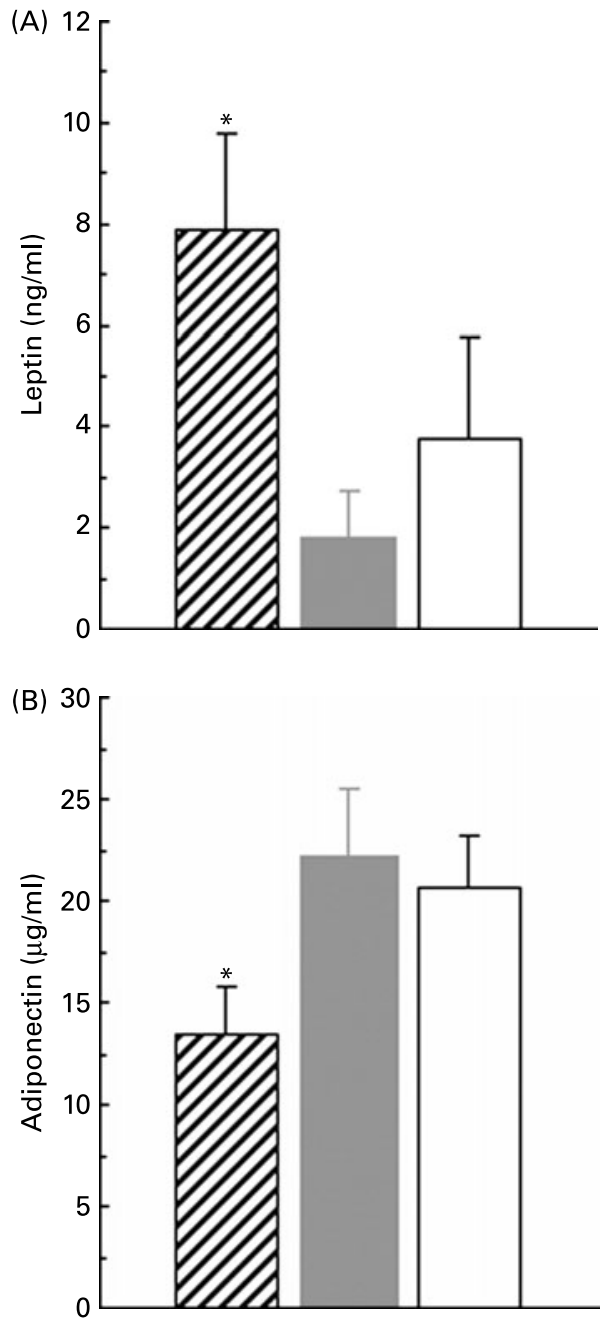

Fig. 3. Plasma glucose (A) and insulin (B) concentration for male CD-1 mice (twenty per group) provided ad libitum access to a high-fat (HF) diet (60\% of energy from fat; $\mathbb{Z}$ ), ad libitum access to a HF diet supplemented with pomegranate seed oil $(61.79 \mathrm{mg} / \mathrm{d}$; HF + POMo; $\square)$ or restricted access to a lowfat diet (lean control; $\square$ ). All measurements were completed after 14 weeks of supplementation. Values are means with their standard errors depicted by vertical bars. Mean values were significantly different from those of the HF + POMo and control groups: ${ }^{*} P<0.05$.

Beyond changes in plasma leptin and adiponectin, we observed that despite a similar energy intake, POMo-supplemented mice gained significantly less total body weight than unsupplemented mice. An interesting anecdotal (not measured, based solely on observation) finding was that the animals which consumed POMo tended to be more active in the later weeks of the study, compared to unsupplemented animals which tended to display sedentary behaviours. In man, consumption of a HF diet is associated with a preference toward sedentary behaviours ${ }^{(21)}$. This has led other researchers to speculate that dietary fat has the ability to act directly or indirectly on the brain to alter behaviour ${ }^{(22,23)}$. The present study was not designed to examine the mechanisms underlying the biological effects of POMo; however, it is reasonable to speculate that POMo may alter the way that dietary fat interacts with the brain. It is equally plausible that POMo may increase fat metabolism and/or energy expenditure, resulting in the attenuation of weight gain that we observed.
More research is needed to mechanistically explore the effect of POMo on pathways that mediate body weight gain.

In addition to changes in leptin and adiponectin, we found that POMo supplementation resulted in the maintenance of fasting insulin concentration at a level similar to lean mice. Despite not detecting a significant difference in blood glucose concentration, it is reasonable to speculate that POMo supplementation resulted in improved insulin sensitivity despite weight gain. An improvement of insulin sensitivity is associated with a decrease in the risk of developing type 2 diabetes. The present finding is consistent with previous reports from our laboratory in $\operatorname{man}^{(4)}$. Leptin has been speculated to play a key role in the mediation of insulin secretion from $\beta$-cells of the pancreas and altering sensitivity ${ }^{(24)}$. Further, insulin and leptin have been reported to have a positive, linear relationship. Since we found a significant leptin difference between $\mathrm{HF}$ and $\mathrm{HF}+\mathrm{POMo}$, this is the most likely reason we observed differences in the insulin concentration. Others have reported that CLA supplementation alters both glucose and insulin ${ }^{(12)}$; however, the present results only partially support this.

Despite reduction in weight gain and type 2 diabetes risk, markers for CVD were not altered. An increase in systemic inflammation is an underlying cause of an increase in CVD risk. In the present study, POMo supplementation did not decrease systemic inflammation, which is the most likely explanation why CVD risk was not changed. Others have reported that when mice are dosed with CLA, a series of anti-inflammatory actions are elicited resulting in a decrease in basal and lipopolysaccharide-stimulated IL-6/ TNF- $\alpha$ production and serum acute phase protein concentration $^{(13,16,17,25)}$. While the present study did not specifically measure inflammatory cytokines (IL-6 and TNF- $\alpha$ ), we did measure two acute-phase proteins (C-reactive protein and haptoglobin), which are generally regarded as more stable biomarkers of systemic inflammatory level ${ }^{(26)}$. Despite a dosage of POMo that was similar to previously reported CLA doses, POMo did not appear to alter systemic inflammation. Since POMo is a naturally occurring source of CLA, it is possible that one must be dosed with more to exert an action similar to synthetic CLA. It is equally plausible that 14 weeks of supplementation was not long enough to elicit changes in markers of CVD risk because they tend to be very stable over time in comparison to markers of type 2 diabetes risk. More research is needed to determine if a different dose of POMo may exert anti-inflammatory effects, similar to those reported for purified CLA. One way to accomplish this would be to individually test the various bioactive compounds found in POMo. It is also possible that lack of significant effects in the present study may have been associated with the absorption of POMo in the digestive track. The present investigation supplemented a HF (60\% of energy from fat) diet with POMo, so it is possible that the POMo had to compete with the other forms of dietary fat for absorption in the digestive tract. Future studies may want to examine the effect of POMo supplementation in weight gain associated with eating a low-fat diet.

In summary, POMo supplementation during 14 weeks of $\mathrm{HF}$ feeding in CD-1 mice resulted in a decrease in total weight gain, leptin and insulin, and an increase in plasma adiponectin concentration. Despite a potential decrease in the risk of developing type 2 diabetes, POMo supplementation did not 
appear to alter CVD risk. It is reasonable to speculate that CVD risk was not altered because POMo lacks the antioxidant properties of pomegranate fruit/juice or was not used at a high enough dose. More research needs to be completed to evaluate fully the potential effects and mechanisms underlying the health benefits of POMo consumption during a period of weight gain.

\section{Acknowledgements}

This study was funded in part by a grant from the Lynda and Stewart Revocable Trust. All animal testing for this research was completed before the end of 2007 with no plans to sponsor future animal testing. The authors do not have any conflict of interest. B. K. M. was responsible for study design, data collection, statistical analysis and manuscript preparation. K. A. S. and M. L. K. were responsible for monitoring animals, data collection/analysis and manuscript preparation. There were no conflicts of interest for any of the authors in this study. The authors would also like to thank Merissa Garvey and Rebecca Kelley for their assistance with managing the mouse colony used in the present study.

\section{References}

1. Christou DD, Gentile CL, DeSouza CA, et al. (2005) Fatness is a better predictor of cardiovascular disease risk factor profile than aerobic fitness in healthy men. Circulation 111, 1904-1914.

2. Fantuzzi G (2005) Adipose tissue, adipokines, and inflammation. J Allergy Clin Immunol 115, 911-919.

3. Hotamisligil GS \& Spiegelman BM (1994) Tumor necrosis factor alpha: a key component of the obesity-diabetes link. Diabetes 43, 1271-1278.

4. McFarlin BK, Johnston CA, Tyler C, et al. (2007) Inflammatory markers are elevated in overweight Mexican-American children. Int J Pediatr Obes 2, 235-241.

5. Gielen S, Adams V, Mobius-Winkler S, et al. (2003) Antiinflammatory effects of exercise training in the skeletal muscle of patients with chronic heart failure. J Am Coll Cardiol 42, 861-868.

6. Greiwe JS, Cheng B, Rubin DC, et al. (2001) Resistance exercise decreases skeletal muscle tumor necrosis factor alpha in frail elderly humans. FASEB J 15, 475-482.

7. McFarlin BK, Flynn MG, Campbell WW, et al. (2006) Physical activity status, but not age, influences inflammatory biomarkers and toll-like receptor 4. J Gerontol A Biol Sci Med Sci 61, 388-393.

8. Smith JK, Dykes R, Douglas JE, et al. (1999) Long-term exercise and atherogenic activity of blood mononuclear cells in persons at risk of developing ischemic heart disease. $J \mathrm{Am}$ Med Assoc 281, 1722-1727.

9. Aviram M, Rosenblat M, Gaitini D, et al. (2004) Pomegranate juice consumption for 3 years by patients with carotid artery stenosis reduces common carotid intima-media thickness, blood pressure and LDL oxidation. Clin Nutr 23, 423-433.
10. Pantuck AJ, Leppert JT, Zomorodian N, et al. (2006) Phase II study of pomegranate juice for men with rising prostate-specific antigen following surgery or radiation for prostate cancer. Clin Cancer Res 12, 4018-4026.

11. Kohno H, Suzuki R, Yasui Y, et al. (2004) Pomegranate seed oil rich in conjugated linolenic acid suppresses chemically induced colon carcinogenesis in rats. Cancer Sci 95, 481-486.

12. Sisk MB, Hausman DB, Martin RJ, et al. (2001) Dietary conjugated linoleic acid reduces adiposity in lean but not obese Zucker rats. J Nutr 131, 1668-1674.

13. Yu Y, Correll PH \& Vanden Heuvel JP (2002) Conjugated linoleic acid decreases production of pro-inflammatory products in macrophages: evidence for a PPAR gamma-dependent mechanism. Biochim Biophys Acta 1581, 89-99.

14. Miller RA \& Nadon NL (2000) Principles of animal use for gerontological research. J Gerontol 55, B117-B123.

15. Fu Y, Luo N, Klein RL, et al. (2005) Adiponectin promotes adipocyte differentiation, insulin sensitivity, and lipid accumulation. J Lipid Res 46, 1369-1379.

16. Changhua L, Jindong Y, Defa L, et al. (2005) Conjugated linoleic acid attenuates the production and gene expression of proinflammatory cytokines in weaned pigs challenged with lipopolysaccharide. J Nutr 135, 239-244.

17. Cheng WL, Lii CK, Chen HW, et al. (2004) Contribution of conjugated linoleic acid to the suppression of inflammatory responses through the regulation of the NF-kappaB pathway. J Agric Food Chem 52, 71-78.

18. Rosenblat M, Hayek $\mathrm{T} \&$ Aviram M (2006) Anti-oxidative effects of pomegranate juice (PJ) consumption by diabetic patients on serum and on macrophages. Atherosclerosis $187,363-371$.

19. Fukumitsu S, Aida K, Ueno N, et al. (2008) Flaxseed lignan attenuates high-fat diet-induced fat accumulation and induces adiponectin expression in mice. Br J Nutr 100, 669-676.

20. Hsu SC \& Huang CJ (2007) Changes in liver PPAR $\alpha$ mRNA expression in response to two levels of high-safflower-oil diets correlate with changes in adiposity and serum leptin in rats and mice. $J$ Nutr Biochem 18, 86-96.

21. Wardle J, Guthrie C, Sanderson S, et al. (2001) Food and activity preferences in children of lean and obese parents. Int $J$ Obes Relat Metab Disord 25, 971-977.

22. Dziedzic B, Szemraj J, Bartkowiak J, et al. (2007) Various dietary fats differentially change the gene expression of neuropeptides involved in body weight regulation in rats. J Neuroendocrinol 19, 364-373.

23. Wang H, Storlien LH \& Huang XF (2002) Effects of dietary fat types on body fatness, leptin, and ARC leptin receptor, NPY, and AgRP mRNA expression. Am J Physiol 282, E1352-E1359.

24. Park S, Hong SM, Sung SR, et al. (2008) Long-term effects of central leptin and resistin on body weight, insulin resistance, and beta-cell function and mass by the modulation of hypothalamic leptin and insulin signaling. Endocrinology 149, 445-454.

25. Browning LM (2003) n-3 Polyunsaturated fatty acids, inflammation and obesity-related disease. Proc Nutr Soc 62, 447-453.

26. de Ferranti SD \& Rifai N (2007) C-reactive protein: a nontraditional serum marker of cardiovascular risk. Cardiovasc Pathol 16, 14-21. 